\title{
Résistance aux antibiotiques: le rôle des patients
}

\author{
Linda Hadorna ${ }^{\text {, Barbara Weil }}{ }^{\mathrm{b}}$ \\ ${ }^{a}$ Collaboratrice scientifique de la division Santé publique, $\mathrm{FMH}_{;}{ }^{\mathrm{b}}$ cheffe de la division Santé publique, FMH
}

Le 3 septembre 1928, Alexandre Fleming découvrit sur ses cultures bactériennes des moisissures qui avaient la propriété d'inhiber leur croissance. Cet évènement marqua la naissance du premier antibiotique moderne. Le développement des antibiotiques constitue l'une des plus grandes avancées de la médecine. Leur utilisation excessive et inappropriée a toutefois pour effet qu'un nombre croissant de bactéries résistent à leur action. Les conséquences sont dramatiques et touchent aussi bien l'être humain que les animaux, l'agriculture et l'environnement - ce qui exige de gérer la problématique selon une approche One Health [1] et de responsabiliser les patients.

Aujourd'hui, les antibiotiques sont devenus incontournables pour la prise en charge médicale des êtres humains et des animaux. Ces médicaments sont indispensables pour traiter les infections bactériennes. Les résistances croissantes constituent toutefois le revers de la médaille. La Suisse et la communauté internationale se doivent d'intervenir. La résistance aux antibiotiques est un problème pour le système de santé, mais également pour l'agriculture et l'environnement.

\section{Commande de la documentation}

La documentation pour les cabinets médicaux, hôpitaux et pharmacies sera présentée dans le cadre d'une rencontre de presse avec I'OFSP, I'OSAV, I'OFAG et l'OFEV le 10 novembre 2017 pendant la Semaine mondiale pour un bon usage des antibiotiques 2017. A partir de cette date, la documentation pourra être commandée gratuitement sur www.bien-utiliser-les-antibiotiques.ch.
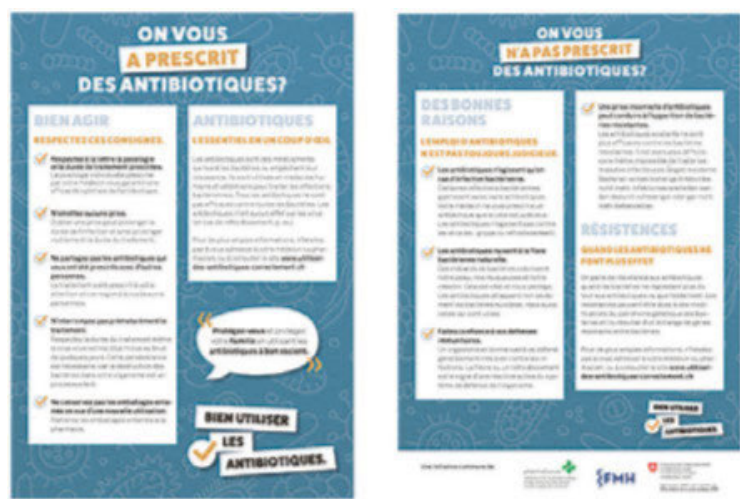

Si nous voulons utiliser efficacement les antibiotiques à l'avenir sans en subir les inconvénients, il faut une action commune et ferme de tous les acteurs concernés. Cette approche dite One Health est également poursuivie dans le cadre de la stratégie nationale Antibiorésistance Suisse (StAR) [2]. Adoptée par le Conseil fédéral le 18 novembre 2015, cette stratégie prévoit pour la Suisse des mesures dans huit domaines d'action stratégiques. Il s'agit des domaines prévention, utilisation rationnelle des antibiotiques, conditions générales, information et formation, coopération, recherche et développement, surveillance et lutte contre la résistance, dans lesquels des mesures seront progressivement mises en œuvre.

Dans le grand public, la question de la résistance aux antibiotiques est abordée sur la base des connaissances disponibles et plus ou moins discutée. Différentes mesures sont prévues pour améliorer l'information du public sur la nature des antibiotiques, leur action, leurs avantages et inconvénients, leur utilisation et la cause des résistances. En effet, la santé se vit au quotidien et dans le contexte socioculturel dans lequel les personnes vivent. La collaboration interprofessionnelle est un outil essentiel pour mettre en œuvre les mesures susmentionnées.

Le présent article met en lumière le thème du point de vue de la médecine humaine. Le deuxième article publié dans ce numéro provient de la Société des vétérinaires suisses. Les deux articles présentent des exemples censés améliorer les connaissances sur la 
résistance aux antibiotiques pour permettre aux spécialistes d'agir de façon responsable et, ce faisant, contribuer à la réduction de la résistance.

\section{Le rôle du patient}

Le patient joue un rôle central, mais les médecins aussi. L'objectif est de responsabiliser chaque individu. Il s'agit de permettre aux personnes, en tant que citoyens et patients, de prendre des décisions, également par rapport à l'utilisation des antibiotiques. Une mesure en rapport avec la lutte contre la résistance aux antibiotiques est la mise à disposition d'informations visant à garantir que

- toutes les personnes puissent trouver et évaluer ces informations,

- toutes les personnes puissent discuter et réfléchir avec d'autres à la question,

- les patients suivent les recommandations et instructions des spécialistes pour la prise ou non-prise d'antibiotiques, et respectent le régime posologique,

- les patients sachent quand ils ont besoin de conseils ou de prestations de santé.

La sensibilisation de chaque individu permet d'obtenir que chacun assume ses responsabilités dans la manière d'utiliser les antibiotiques et prenne ses décisions en connaissance de cause. Dans ce contexte, les interfaces entre environnement, individus et spécialistes sont déterminantes. Pour renforcer la compétence en matière de santé des patients traités aux antibiotiques et mettre une documentation à disposition des médecins prescripteurs et pharmaciens délivrant les antibiotiques, des messages clés concernant l'utilisation des antibiotiques ont été élaborés.

\section{Documentation pour les patients - faire bon usage des antibiotiques}

Correspondance: FMH - Fédération des médecins suisses

Département Santé publique Elfenstrasse 18

Case postale 300

CH-3000 Berne 15

public.health[at]fmh.ch
La documentation pour les patients vise à interpeller ceux-ci lors de la remise d'antibiotiques et à les sensibiliser au comportement correct en cas de remise ou non-remise d'antibiotiques.

Afin de saisir les critères centraux et messages clés de la documentation pour les patients consacrée à la résis- tance aux antibiotiques, les éléments prioritaires ont été déterminés à l'aide du charting (saisie structurée d'éléments clés) et collating (tri, systématisation, catégorisation, résumé et écriture des résultats). Le collating a été réalisé par des médecins et spécialistes de l'OFSP. Les messages clés incluent la prescription obligatoire, le mode d'action, l'observance, la posologie, les risques d'une utilisation excessive et les options thérapeutiques. La documentation pour les patients a été adaptée au groupe-cible, clairement structurée, rédigée dans un langage compréhensible et adéquatement illustrée. La pertinence scientifique des messages délivrés est au cœur de tous les thèmes.

\section{Combattre ensemble la résistance aux antibiotiques}

Les canaux d'information adéquats pour les personnes recevant des antibiotiques ont été définis en commun par l'Office fédéral de la santé publique (OFSP), la Fédération des médecins suisses (FMH) et la Société suisse des pharmaciens (pharmaSuisse), en étroite collaboration avec une agence de communication spécialisée dans la communication agile. Deux groupes de réflexion composés de médecins de la Société suisse de gynécologie et obstétrique, de la Société suisse de pédiatrie, du Collège de médecine de premier recours, de la SSMIG et d'experts du domaine de l'infectiologie ont retravaillé la documentation. La collaboration interprofessionnelle a produit une documentation qui permettra, grâce à une utilisation commune, de contribuer à la lutte contre la résistance aux antibiotiques.

L'approche globale a pour objectif d'apporter à tous les patients les mêmes messages fondés sur les preuves et d'assurer que l'information soit facilement reconnaissable. En utilisant la documentation pour les patients lorsqu'ils communiquent avec eux, les médecins contribuent à ce que ceux-ci puissent assumer leur part de responsabilité conformément à l'approche One Health.

\section{Références}

1 http://www onehealthinitiative.com

2 Stratégie nationale Antibiorésistance (StAR), https://www.star admin ch 